\title{
Interactions between a massless tensor field with the mixed symmetry of the Riemann tensor and a massless vector field
}

\author{
C. Bizdadea*, C. C. Ciobîrcăł E. M. Cioroianu \\ Faculty of Physics, University of Craiova \\ 13 A. I. Cuza Str., Craiova 200585, Romania
}

December 19, 2018

\begin{abstract}
Consistent couplings between a massless tensor field with the mixed symmetry of the Riemann tensor and a massless vector field are analyzed in the framework of Lagrangian BRST cohomology. Under the assumptions on smoothness, locality, Lorentz covariance, and Poincaré invariance of the deformations, combined with the requirement that the interacting Lagrangian is at most second-order derivative, it is proved that there are no consistent cross-interactions between a single massless tensor field with the mixed symmetry of the Riemann tensor and one massless vector field.
\end{abstract}

PACS number: 11.10.Ef

\section{Introduction}

Mixed symmetry type tensor fields [1]-[7] are involved in many physically interesting theories, like superstrings, supergravities, or supersymmetric high

\footnotetext{
*e-mail address: bizdadea@central.ucv.ro

$\dagger$ e-mail address: ciobarca@central.ucv.ro

†e-mail address: manache@central.ucv.ro

§e-mail address: osaliu@central.ucv.ro
} 
spin theories. The study of gauge theories with mixed symmetry type tensor fields revealed several issues, like the dual formulation of field theories of spin two or higher [8]-[15], the impossibility of consistent interactions in the dual formulation of linearized gravity [16], or a Lagrangian first-order approach [12, 17, 18] to some classes of free massless mixed symmetry type tensor gauge fields, suggestively resembling to the tetrad formalism of General Relativity. One of the most important aspects related to this type of gauge models is the analysis of their consistent interactions, among themselves as well as with higher-spin gauge theories [19]-[28]. The best approach to this matter is the cohomological one, based on the deformation of the solution to the master equation [29]. The aim of our paper is to investigate the manifestly covariant consistent interactions between a single, free, massless tensor gauge field $t_{\mu \nu \mid \alpha \beta}$ with the mixed symmetry of the Riemann tensor and a massless vector field.

Our procedure relies on the deformation of the solution to the master equation by means of local BRST cohomology. For each situation, we initially determine the associated free antifield-BRST symmetry $s$, which splits as the sum between the Koszul-Tate differential and the exterior longitudinal derivative only, $s=\delta+\gamma$. Then, we solve the basic equations of the deformation procedure. Under the supplementary assumptions on smoothness, locality, Lorentz covariance, and Poincaré invariance of the deformations as well as on the maximum derivative order of the interacting Lagrangian being equal to two, we prove that there are no consistent cross-interactions between the tensor field with the mixed symmetry of the Riemann tensor and the massless vector field.

The paper is organized in four sections. Section 2 is focused on the presentation of the free model under study and on the construction of the associated BRST differential. In Section 3 we briefly review the antifieldBRST deformation procedure. Section 4 analyzes the consistent couplings between the tensor field with the mixed symmetry of the Riemann tensor and the massless vector field with the help of the local BRST cohomology of the free model. Section 5 ends the paper with some conclusions. 


\section{Free model. Free BRST symmetry}

The starting point is given by the free Lagrangian action

$$
\begin{aligned}
S_{0}\left[t_{\mu \nu \mid \alpha \beta}, A_{\mu}\right]= & \int d^{D} x\left[\frac{1}{8}\left(\partial^{\lambda} t^{\mu \nu \mid \alpha \beta}\right)\left(\partial_{\lambda} t_{\mu \nu \mid \alpha \beta}\right)-\left(\partial_{\mu} t^{\mu \nu \mid \alpha \beta}\right)\left(\partial_{\beta} t_{\nu \alpha}\right)\right. \\
& -\frac{1}{2}\left(\partial_{\mu} t^{\mu \nu \mid \alpha \beta}\right)\left(\partial^{\lambda} t_{\lambda \nu \mid \alpha \beta}\right)-\frac{1}{2}\left(\partial^{\lambda} t^{\nu \beta}\right)\left(\partial_{\lambda} t_{\nu \beta}\right) \\
& +\left(\partial_{\nu} t^{\nu \beta}\right)\left(\partial^{\lambda} t_{\lambda \beta}\right)-\frac{1}{2}\left(\partial_{\nu} t^{\nu \beta}\right)\left(\partial_{\beta} t\right) \\
& \left.+\frac{1}{8}\left(\partial^{\lambda} t\right)\left(\partial_{\lambda} t\right)-\frac{1}{4} F_{\mu \nu} F^{\mu \nu}\right] \equiv S_{0}^{\mathrm{t}}\left[t_{\mu \nu \mid \alpha \beta}\right]+S_{0}^{\mathrm{A}}\left[A_{\mu}\right](1)
\end{aligned}
$$

in a Minkowski-flat space-time of dimension $D \geq 5$, endowed with a metric tensor of 'mostly plus' signature $\sigma_{\mu \nu}=\sigma^{\mu \nu}=(-++++\cdots)$. The massless tensor field $t_{\mu \nu \mid \alpha \beta}$ of rank four has the mixed symmetry of the Riemann tensor and hence transforms according to an irreducible representation of $G L(D, \mathbb{R})$ corresponding to a rectangular Young diagram with two columns and two rows. Thus, it is separately antisymmetric in the pairs $\{\mu, \nu\}$ and $\{\alpha, \beta\}$, is symmetric under the interchange of these pairs $(\{\mu, \nu\} \longleftrightarrow\{\alpha, \beta\})$, and satisfies the identity $t_{[\mu \nu \mid \alpha] \beta} \equiv 0$ associated with the above diagram, which we will refer to as the Bianchi I identity. Here and in the sequel the symbol $[\mu \nu \cdots]$ signifies complete antisymmetry with respect to the (Lorentz) indices between brackets, with the conventions that the minimum number of terms is always used and the result is never divided by the number of terms. (For instance, we have that $t_{[\mu \nu \mid \alpha] \beta}=t_{\mu \nu \mid \alpha \beta}+t_{\nu \alpha \mid \mu \beta}+t_{\alpha \mu \mid \nu \beta}$.) The notation $t_{\nu \beta}$ signifies the simple trace of the original tensor field, $t_{\nu \beta}=\sigma^{\mu \alpha} t_{\mu \nu \mid \alpha \beta}$, which is symmetric, $t_{\nu \beta}=t_{\beta \nu}$, while $t$ denotes its double trace, $t=\sigma^{\nu \beta} t_{\nu \beta} \equiv t^{\mu \nu}{ }_{\mid \mu \nu}$, which is a scalar. A generating set of gauge transformations for the action (1) reads as

$$
\delta_{\epsilon} t_{\mu \nu \mid \alpha \beta}=\partial_{\mu} \epsilon_{\alpha \beta \mid \nu}-\partial_{\nu} \epsilon_{\alpha \beta \mid \mu}+\partial_{\alpha} \epsilon_{\mu \nu \mid \beta}-\partial_{\beta} \epsilon_{\mu \nu \mid \alpha}, \quad \delta_{\epsilon} A_{\mu}=\partial_{\mu} \epsilon,
$$

with the bosonic gauge parameters $\epsilon_{\mu \nu \mid \alpha}$ transforming according to an irreducible representation of $G L(D, \mathbb{R})$ corresponding to a three-cell Young diagram with two columns and two rows (also known as a hook diagram), being therefore antisymmetric in the pair $\mu \nu$ and satisfying the identity $\epsilon_{[\mu \nu \mid \alpha]} \equiv 0$. The last identity is required in order to ensure that the gauge transformations (2) check the same Bianchi I identity like the fields themselves, namely, 
$\delta_{\epsilon} t_{[\mu \nu \mid \alpha] \beta} \equiv 0$. The above generating set of gauge transformations is Abelian and off-shell, first-stage reducible since if we make the transformation

$$
\epsilon_{\mu \nu \mid \alpha}=2 \partial_{\alpha} \theta_{\mu \nu}-\partial_{[\mu} \theta_{\nu] \alpha},
$$

with $\theta_{\mu \nu}$ an arbitrary antisymmetric tensor $\left(\theta_{\mu \nu}=-\theta_{\nu \mu}\right)$, then the gauge transformations of the tensor field identically vanish, $\delta_{\epsilon(\theta)} t_{\mu \nu \mid \alpha \beta} \equiv 0$.

In agreement with the general setting of the antibracket-antifield formalism, the construction of the BRST symmetry for the free theory under consideration starts with the identification of the BRST algebra on which the BRST differential $s$ acts. The generators of the BRST algebra are of two kinds: fields/ghosts and antifields. The ghost spectrum for the model under study comprises the fermionic ghosts $\eta_{\alpha \beta \mid \mu}$ and $\eta$ associated with the gauge parameters $\epsilon_{\alpha \beta \mid \mu}$ and $\epsilon$ from (2) as well as the bosonic ghosts for ghosts $C_{\mu \nu}$ due to the first-stage reducibility parameters $\theta_{\mu \nu}$ in (3). In order to make compatible the behaviour of $\epsilon_{\alpha \beta \mid \mu}$ and $\theta_{\mu \nu}$ with that of the corresponding ghosts, we ask that $\eta_{\alpha \beta \mid \mu}$ satisfies the properties $\eta_{\mu \nu \mid \alpha}=-\eta_{\nu \mu \mid \alpha}, \eta_{[\mu \nu \mid \alpha]} \equiv 0$ and that $C_{\mu \nu}$ is antisymmetric. The antifield spectrum is organized into the antifields $t^{* \mu \nu \mid \alpha \beta}$ and $A^{* \mu}$ of the original tensor fields and those of the ghosts, $\eta^{* \mu \nu \mid \alpha}, \eta^{*}$, and $C^{* \mu \nu}$, of statistics opposite to that of the associated fields/ghosts. It is understood that $t^{* \mu \nu \mid \alpha \beta}$ is subject to the conditions

$$
t^{* \mu \nu \mid \alpha \beta}=-t^{* \nu \mu \mid \alpha \beta}=-t^{* \mu \nu \mid \beta \alpha}=t^{* \alpha \beta \mid \mu \nu}, \quad t^{*[\mu \nu \mid \alpha] \beta} \equiv 0
$$

and, along the same line, it is required that

$$
\eta^{* \mu \nu \mid \alpha}=-\eta^{* \nu \mu \mid \alpha}, \quad \eta^{*[\mu \nu \mid \alpha]} \equiv 0, \quad C^{* \mu \nu}=-C^{* \nu \mu} .
$$

We will denote the simple and double traces of $t^{* \mu \nu \mid \alpha \beta}$ by

$$
t^{* \nu \beta}=\sigma_{\mu \alpha} t^{* \mu \nu \mid \alpha \beta}, \quad t^{*}=\sigma_{\nu \beta} t^{* \nu \beta},
$$

such that $t^{* \nu \beta}$ is symmetric and $t^{*}$ is a scalar.

As both the gauge generators and reducibility functions for this model are field-independent, it follows that the associated BRST differential $\left(s^{2}=0\right)$ splits into

$$
s=\delta+\gamma,
$$

where $\delta$ represents the Koszul-Tate differential $\left(\delta^{2}=0\right)$, graded by the antighost number agh $(\operatorname{agh}(\delta)=-1)$, and $\gamma$ stands for the exterior derivative along the gauge orbits. It turns out to be a true differential $\left(\gamma^{2}=0\right)$ 
that anticommutes with $\delta(\delta \gamma+\gamma \delta=0)$, whose degree is named pure ghost number $\operatorname{pgh}(\operatorname{pgh}(\gamma)=1)$. These two degrees do not interfere $(\operatorname{agh}(\gamma)=0$, $\operatorname{pgh}(\delta)=0)$. The overall degree that grades the BRST differential is known as the ghost number (gh) and is defined like the difference between the pure ghost number and the antighost number, such that $\operatorname{gh}(s)=\operatorname{gh}(\delta)=\operatorname{gh}(\gamma)=$ 1. According to the standard rules of the BRST method, the corresponding degrees of the generators from the BRST complex are valued like

$$
\begin{aligned}
\operatorname{pgh}\left(t_{\mu \nu \mid \alpha \beta}\right) & =0=\operatorname{pgh}\left(A_{\mu}\right), \\
\operatorname{pgh}\left(\eta_{\mu \nu \mid \alpha}\right) & =1=\operatorname{pgh}(\eta), \quad \operatorname{pgh}\left(C_{\mu \nu}\right)=2, \\
\operatorname{pgh}\left(t^{* \mu \nu \mid \alpha \beta}\right) & \left.=\operatorname{pgh}\left(A^{* \mu}\right)=\operatorname{pgh}\left(\eta^{* \mu \nu \mid \alpha}\right)=\operatorname{pgh}\left(\eta^{*}\right)=\operatorname{pgh}\left(C^{* \mu \nu}\right)=010\right) \\
\operatorname{agh}\left(t_{\mu \nu \mid \alpha \beta}\right) & =\operatorname{agh}\left(A_{\mu}\right)=\operatorname{agh}\left(\eta_{\mu \nu \mid \alpha}\right)=\operatorname{agh}(\eta)=\operatorname{agh}\left(C_{\mu \nu}\right)=0, \\
\operatorname{agh}\left(t^{* \mu \nu \mid \alpha \beta}\right) & =1=\operatorname{agh}\left(A^{* \mu}\right), \\
\operatorname{agh}\left(\eta^{* \mu \nu \mid \alpha}\right) & =2=\operatorname{agh}\left(\eta^{*}\right), \quad \operatorname{agh}\left(C^{* \mu \nu}\right)=3,
\end{aligned}
$$

and the actions of $\delta$ and $\gamma$ on them are given by

$$
\begin{aligned}
\gamma t_{\mu \nu \mid \alpha \beta} & =\partial_{\mu} \eta_{\alpha \beta \mid \nu}-\partial_{\nu} \eta_{\alpha \beta \mid \mu}+\partial_{\alpha} \eta_{\mu \nu \mid \beta}-\partial_{\beta} \eta_{\mu \nu \mid \alpha} \\
\gamma \eta_{\mu \nu \mid \alpha} & =2 \partial_{\alpha} C_{\mu \nu}-\partial_{[\mu} C_{\nu] \alpha}, \quad \gamma C_{\mu \nu}=0 \\
\gamma t^{* \mu \nu \mid \alpha \beta} & =\gamma \eta^{* \mu \nu \mid \alpha}=\gamma C^{* \mu \nu}=0 \\
\delta t_{\mu \nu \mid \alpha \beta} & =\delta \eta_{\mu \nu \mid \alpha}=\delta C_{\mu \nu}=0 \\
\delta t^{* \mu \nu \mid \alpha \beta} & =\frac{1}{4} T^{\mu \nu \mid \alpha \beta}, \quad \delta \eta^{* \alpha \beta \mid \nu}=-4 \partial_{\mu} t^{* \mu \nu \mid \alpha \beta}, \quad \delta C^{* \mu \nu}=3 \partial_{\alpha} \eta^{* \mu \nu \mid \alpha}, \\
\gamma A_{\mu} & =\partial_{\mu} \eta, \quad \gamma \eta=0, \quad \gamma A^{* \mu}=0, \quad \gamma \eta^{*}=0, \\
\delta A_{\mu} & =0, \quad \delta \eta=0, \quad \delta A^{* \mu}=-\partial_{\nu} F^{\mu \nu}, \quad \delta \eta^{*}=-\partial_{\mu} A^{* \mu},
\end{aligned}
$$

with $T_{\mu \nu \mid \alpha \beta}$ of the form

$$
\begin{aligned}
T_{\mu \nu \mid \alpha \beta}= & \square t_{\mu \nu \mid \alpha \beta}+\partial^{\rho}\left(\partial_{\mu} t_{\alpha \beta \mid \nu \rho}-\partial_{\nu} t_{\alpha \beta \mid \mu \rho}+\partial_{\alpha} t_{\mu \nu \mid \beta \rho}-\partial_{\beta} t_{\mu \nu \mid \alpha \rho}\right) \\
& +\left(\partial_{\mu} \partial_{\alpha} t_{\beta \nu}-\partial_{\mu} \partial_{\beta} t_{\alpha \nu}-\partial_{\nu} \partial_{\alpha} t_{\beta \mu}+\partial_{\nu} \partial_{\beta} t_{\alpha \mu}\right) \\
& -\frac{1}{2} \partial^{\lambda} \partial^{\rho}\left(\sigma_{\mu \alpha}\left(t_{\lambda \beta \mid \nu \rho}+t_{\lambda \nu \mid \beta \rho}\right)-\sigma_{\mu \beta}\left(t_{\lambda \alpha \mid \nu \rho}+t_{\lambda \nu \mid \alpha \rho}\right)\right. \\
& \left.-\sigma_{\nu \alpha}\left(t_{\lambda \beta \mid \mu \rho}+t_{\lambda \mu \mid \beta \rho}\right)+\sigma_{\nu \beta}\left(t_{\lambda \alpha \mid \mu \rho}+t_{\lambda \mu \mid \alpha \rho}\right)\right) \\
& -\square\left(\sigma_{\mu \alpha} t_{\beta \nu}-\sigma_{\mu \beta} t_{\alpha \nu}-\sigma_{\nu \alpha} t_{\beta \mu}+\sigma_{\nu \beta} t_{\alpha \mu}\right) \\
& +\partial^{\rho}\left(\sigma_{\mu \alpha}\left(\partial_{\beta} t_{\nu \rho}+\partial_{\nu} t_{\beta \rho}\right)-\sigma_{\mu \beta}\left(\partial_{\alpha} t_{\nu \rho}+\partial_{\nu} t_{\alpha \rho}\right)\right. \\
& \left.-\sigma_{\nu \alpha}\left(\partial_{\beta} t_{\mu \rho}+\partial_{\mu} t_{\beta \rho}\right)+\sigma_{\nu \beta}\left(\partial_{\alpha} t_{\mu \rho}+\partial_{\mu} t_{\alpha \rho}\right)\right)
\end{aligned}
$$




$$
\begin{aligned}
& -\frac{1}{2}\left(\sigma_{\mu \alpha} \partial_{\beta} \partial_{\nu}-\sigma_{\mu \beta} \partial_{\alpha} \partial_{\nu}-\sigma_{\nu \alpha} \partial_{\beta} \partial_{\mu}+\sigma_{\nu \beta} \partial_{\alpha} \partial_{\mu}\right) t \\
& -\left(\sigma_{\mu \alpha} \sigma_{\nu \beta}-\sigma_{\mu \beta} \sigma_{\nu \alpha}\right)\left(\partial^{\lambda} \partial^{\rho} t_{\lambda \rho}-\frac{1}{2} \square t\right) .
\end{aligned}
$$

Both $\delta$ and $\gamma$ (and implicitly $s$ ) were taken to act like right derivations.

The antifield-BRST differential is known to admit a canonical action in a structure named antibracket and defined by decreeing the fields/ghosts conjugated with the corresponding antifields, $s \cdot=(\cdot, S)$, where $($,$) signifies the$ antibracket and $S$ denotes the canonical generator of the BRST symmetry. It is a bosonic functional of ghost number zero involving both the field/ghost and antifield spectra, which obeys the classical master equation

$$
(S, S)=0
$$

The classical master equation is equivalent with the second-order nilpotency of $s, s^{2}=0$, while its solution encodes the entire gauge structure of the associated theory. Taking into account the formulas (14)-(20) as well as the actions of $\delta$ and $\gamma$ in canonical form, we find that the complete solution to the master equation for the model under study reads as

$$
\begin{aligned}
S= & S_{0}\left[t_{\mu \nu \mid \alpha \beta}, A_{\mu}\right]+\int d^{D} x\left[t ^ { * \mu \nu | \alpha \beta } \left(\partial_{\mu} \eta_{\alpha \beta \mid \nu}-\partial_{\nu} \eta_{\alpha \beta \mid \mu}+\partial_{\alpha} \eta_{\mu \nu \mid \beta}\right.\right. \\
& \left.\left.-\partial_{\beta} \eta_{\mu \nu \mid \alpha}\right)+\eta^{* \mu \nu \mid \alpha}\left(2 \partial_{\alpha} C_{\mu \nu}-\partial_{[\mu} C_{\nu] \alpha}\right)+A^{* \mu} \partial_{\mu} \eta\right] .
\end{aligned}
$$

The main ingredients of the antifield-BRST symmetry derived in this section will be useful in the sequel at the analysis of consistent interactions that can be added to the action (1) without changing its number of independent gauge symmetries.

\section{Brief review of the antifield-BRST defor- mation procedure}

There are three main types of consistent interactions that can be added to a given gauge theory: the first type deforms only the Lagrangian action, but not its gauge transformations, the second kind modifies both the action and its transformations, but not the gauge algebra, and the third, and certainly most interesting category, changes everything, namely, the action, its gauge symmetries, and the accompanying algebra. 
The reformulation of the problem of consistent deformations of a given action and of its gauge symmetries in the antifield-BRST setting is based on the observation that if a deformation of the classical theory can be consistently constructed, then the solution to the master equation for the initial theory can be deformed into

$$
\bar{S}=S+g S_{1}+g^{2} S_{2}+O\left(g^{3}\right), \quad \varepsilon(\bar{S})=0, \quad \operatorname{gh}(\bar{S})=0,
$$

such that

$$
(\bar{S}, \bar{S})=0
$$

Here and in the sequel $\varepsilon(F)$ denotes the Grassmann parity of $F$. The projection of (25) on the various powers in the coupling constant induces the following tower of equations:

$$
\begin{array}{ll}
g^{0}:(S, S)=0 \\
g^{1}:\left(S_{1}, S\right)=0 \\
g^{2}: \frac{1}{2}\left(S_{1}, S_{1}\right)+\left(S_{2}, S\right)=0,
\end{array}
$$

The first equation is satisfied by hypothesis. The second one governs the first-order deformation of the solution to the master equation $\left(S_{1}\right)$ and it shows that $S_{1}$ is a BRST co-cycle, $s S_{1}=0$. This means that $S_{1}$ pertains to the ghost number zero cohomological space of $s, H^{0}(s)$, which is generically non-empty due to its isomorphism to the space of physical observables of the free theory. The remaining equations are responsible for the higher-order deformations of the solution to the master equation. No obstructions arise in finding solutions to them as long as no further restrictions, such as spacetime locality, are imposed. Obviously, only nontrivial first-order deformations should be considered, since trivial ones $\left(S_{1}=s B\right)$ lead to trivial deformations of the initial theory and can be eliminated by convenient redefinitions of the fields. Ignoring the trivial deformations, it follows that $S_{1}$ is a nontrivial BRST-observable, $S_{1} \in H^{0}(s)$. Once that the deformation equations (27)(28), etc., have been solved by means of specific cohomological techniques, from the consistent nontrivial deformed solution to the master equation we can extract all the information on the gauge structure of the accompanying interacting theory. 


\section{First-order deformation}

The purpose of our paper is to study the consistent interactions that can be added to the free action (1) by means of solving the main deformation equations, namely, (27)-(28), etc. For obvious reasons, we consider only smooth, local, Lorentz-covariant, and Poincaré-invariant deformations. If we make the notation $S_{1}=\int d^{D} x a$, with $a$ a local function, then the local form of the equation (27), which we have seen that controls the first-order deformation of the solution to the master equation, becomes

$$
s a=\partial_{\mu} m^{\mu}, \quad \operatorname{gh}(a)=0, \quad \varepsilon(a)=0,
$$

for some $m^{\mu}$, and it shows that the nonintegrated density of the first-order deformation pertains to the local cohomology of $s$ at ghost number zero, $a \in H^{0}(s \mid d)$, where $d$ denotes the exterior space-time differential. In order to analyze the above equation, we develop $a$ according to the antighost number

$$
a=\sum_{k=0}^{I} a_{k}, \quad \operatorname{agh}\left(a_{k}\right)=k, \quad \operatorname{gh}\left(a_{k}\right)=0, \quad \varepsilon\left(a_{k}\right)=0,
$$

and assume, without loss of generality, that $a$ stops at some finite value $I$ of the antighost number 1 By taking into account the decomposition (7) of the BRST differential, the equation (29) is equivalent to a tower of local equations, corresponding to the various decreasing values of the antighost number

$$
\begin{aligned}
\gamma a_{I} & =\partial_{\mu} \stackrel{(I)}{m}^{\mu} \\
\delta a_{I}+\gamma a_{I-1} & =\partial_{\mu} \stackrel{(I-1)}{m}^{\mu}, \\
\delta a_{k}+\gamma a_{k-1} & =\partial_{\mu}{\stackrel{(k-1)^{\mu}}{m}}^{\mu}, \quad I-1 \geq k \geq 1,
\end{aligned}
$$

where $\left(\begin{array}{l}(k)^{\mu} \\ m\end{array}\right)_{k=\overline{0, I}}$ are some local currents, with agh $\left(\stackrel{(k)}{m}^{\mu}\right)=k$. It can be proved $^{2}$ that one can replace the equation (31) at strictly positive antighost

\footnotetext{
${ }^{1}$ This can be shown, for instance, like in [31] (Section 3) or [32, under the sole assumption that the interacting Lagrangian at the first order in the coupling constant, $a_{0}$, has a finite, but otherwise arbitrary derivative order.

${ }^{2}$ The fact that it is possible to replace the equation (31) with (34) can be done like in the proof of Corollary 3 from 33, with the precaution to include in an appropriate manner the dependence on the vector field BRST sector.
} 
numbers with

$$
\gamma a_{I}=0, \quad I>0 .
$$

In conclusion, under the assumption that $I>0$, the representative of highest antighost number from the nonintegrated density of the first-order deformation can always be taken to be $\gamma$-closed, such that the equation (29) associated with the local form of the first-order deformation is completely equivalent to the tower of equations (34) and (32)-(33).

Before proceeding to the analysis of the solutions to the first-order deformation equation, we briefly comment on the uniqueness and triviality of such

solutions. Due to the second-order nilpotency of $\gamma\left(\gamma^{2}=0\right)$, the solution to the top equation (34) is clearly unique up to $\gamma$-exact contributions,

$$
a_{I} \rightarrow a_{I}+\gamma b_{I}, \quad \operatorname{agh}\left(b_{I}\right)=I, \quad \operatorname{pgh}\left(b_{I}\right)=I-1, \quad \varepsilon\left(b_{I}\right)=1 .
$$

Meanwhile, if it turns out that $a_{I}$ reduces to $\gamma$-exact terms only, $a_{I}=\gamma b_{I}$, then it can be made to vanish, $a_{I}=0$. In other words, the nontriviality of the first-order deformation $a$ is translated at its highest antighost number component into the requirement that

$$
a_{I} \in H^{I}(\gamma)
$$

where $H^{I}(\gamma)$ denotes the cohomology of the exterior longitudinal derivative $\gamma$ at pure ghost number equal to $I$. At the same time, the general condition on the nonintegrated density of the first-order deformation to be in a nontrivial cohomological class of $H^{0}(s \mid d)$ shows on the one hand that the solution to (29) is unique up to $s$-exact pieces plus total divergences

$$
a \rightarrow a+s b+\partial_{\mu} n^{\mu}, \quad \operatorname{gh}(b)=-1, \quad \varepsilon(b)=1, \quad \operatorname{gh}\left(n^{\mu}\right)=0, \quad \varepsilon\left(n^{\mu}\right)=0
$$

and on the other hand that if the general solution to (29) is found to be completely trivial, $a=s b+\partial_{\mu} n^{\mu}$, then it can be made to vanish, $a=0$.

\subsection{Basic cohomologies}

In the light of the above discussion, we pass to the investigation of the solutions to the equations (34) and (32)-(33). We have seen that $a_{I}$ belongs to the cohomology of the exterior longitudinal derivative (see the formula (36)), such that we need to compute $H(\gamma)$ in order to construct the component of 
highest antighost number from the first-order deformation. This matter is solved with the help of the definitions (14)-(16) and (19).

In order to determine the cohomology $H(\gamma)$, we split the differential $\gamma$ into two pieces

$$
\gamma=\gamma_{t}+\gamma_{A},
$$

where $\gamma_{t}$ acts nontrivially only on the fields/ghosts from the $t_{\mu \nu \mid \alpha \beta}$ sector and $\gamma_{A}$ does the same thing, but with respect to the vector field sector. From the above splitting it follows that the nilpotency of $\gamma$ is equivalent to the nilpotency and anticommutativity of its components

$$
\left(\gamma_{t}\right)^{2}=0=\left(\gamma_{A}\right)^{2}, \quad \gamma_{t} \gamma_{A}+\gamma_{A} \gamma_{t}=0
$$

Kunneth's formula then ensures the isomorphism

$$
H(\gamma)=H\left(\gamma_{t}\right) \otimes H\left(\gamma_{A}\right)
$$

Thus, we can state that $H(\gamma)$ is generated [36] on the one hand by $\chi^{* \Delta}, F_{\mu \nu}$, and $F_{\mu \nu \lambda \mid \alpha \beta \gamma}$ as well as by their space-time derivatives and on the other hand by the ghosts $C_{\mu \nu}, \partial_{[\mu} C_{\nu] \alpha}$, and $\eta$, where $\chi^{* \Delta}$ is a collective notation for all the antifields

$$
\chi^{* \Delta}=\left\{t^{* \mu \nu \mid \alpha \beta}, A^{* \mu}, \eta^{* \mu \nu \mid \alpha}, \eta^{*}, C^{* \mu \nu}\right\},
$$

while

$$
\begin{aligned}
F_{\mu \nu \lambda \mid \alpha \beta \gamma}= & \partial_{\lambda} \partial_{\gamma} t_{\mu \nu \mid \alpha \beta}+\partial_{\mu} \partial_{\gamma} t_{\nu \lambda \mid \alpha \beta}+\partial_{\nu} \partial_{\gamma} t_{\lambda \mu \mid \alpha \beta} \\
& +\partial_{\lambda} \partial_{\alpha} t_{\mu \nu \mid \beta \gamma}+\partial_{\mu} \partial_{\alpha} t_{\nu \lambda \mid \beta \gamma}+\partial_{\nu} \partial_{\alpha} t_{\lambda \mu \mid \beta \gamma} \\
& +\partial_{\lambda} \partial_{\beta} t_{\mu \nu \mid \gamma \alpha}+\partial_{\mu} \partial_{\beta} t_{\nu \lambda \mid \gamma \alpha}+\partial_{\nu} \partial_{\beta} t_{\lambda \mu \mid \gamma \alpha},
\end{aligned}
$$

represent the components of the curvature tensor for $t_{\mu \nu \mid \alpha \beta}$ (the quantities with the minimum number of derivatives, invariant under the gauge transformations $\delta_{\epsilon} t_{\mu \nu \mid \alpha \beta}$ in (2) ). (The quantity $\partial_{[\mu} C_{\nu] \alpha}$ carries a trivial component. Its non-trivial part is given by the completely antisymmetric expression $\partial_{[\mu} C_{\nu \alpha]}$, which differs from our representative by a $\gamma$-exact term.) So, the most general (and nontrivial), local solution to (34) can be written, up to $\gamma$-exact contributions, as

$$
a_{I}=\alpha_{I}\left(\left[F_{\mu \nu}\right],\left[F_{\mu \nu \lambda \mid \alpha \beta \gamma}\right],\left[\chi^{* \Delta}\right]\right) \omega^{I}\left(C_{\mu \nu}, \partial_{[\mu} C_{\nu] \alpha}, \eta\right)
$$

where the notation $f([q])$ means that $f$ depends on $q$ and its derivatives up to a finite order and $\omega^{I}$ denotes the elements of a basis in the space 
of polynomials with pure ghost number $I$ in the corresponding ghosts and some of their first-order derivatives. The objects $\alpha_{I}$ (obviously nontrivial in $H^{0}(\gamma)$ ) were taken to have a bounded number of derivatives and therefore they are polynomials in the antifields $\chi^{* \Delta}$, in $F_{\mu \nu}$, in the curvature tensor $F_{\mu \nu \lambda \mid \alpha \beta \gamma}$ as well as in their derivatives. Due to the fact that these elements are $\gamma$-closed, they are called invariant polynomials. At zero antighost number, the invariant polynomials are polynomials in the curvature tensor $F_{\mu \nu \lambda \mid \alpha \beta \gamma}$, the field strength $F_{\mu \nu}$, and their derivatives.

Replacing the solution (43) in the equation (32), we remark that a necessary (but not sufficient) condition for the existence of (nontrivial) solutions $a_{I-1}$ is that the invariant polynomials $\alpha_{I}$ from (43) are (nontrivial) objects from the local cohomology of the Koszul-Tate differential $H(\delta \mid d)$ at antighost number $I>0$ and pure ghost number equal to zerd 3 , $\alpha_{I} \in H_{I}(\delta \mid d)$, i.e.

$$
\delta \alpha_{I}=\partial_{\mu} j^{\mu}, \quad \varepsilon\left(j^{\mu}\right)=1, \quad \operatorname{agh}\left(j^{\mu}\right)=I-1, \quad \operatorname{pgh}\left(j^{\mu}\right)=0 .
$$

(In view of the footnote 3 from now on it is understood that by $H_{I}(\delta \mid d)$ we mean the local cohomology of the Koszul-Tate differential at antighost $I$ and at pure ghost number zero.) Consequently, we need to investigate some of the main properties of the local cohomology of the Koszul-Tate differential at strictly positive antighost numbers in order to completely determine the component $a_{I}$ of highest antighost number in the first-order deformation. As the free model under study is a normal gauge theory of Cauchy order equal to three, the general results from [34, 35] ensure that the local cohomology of the Koszul-Tate differential is trivial at antighost numbers strictly greater than its Cauchy order

$$
H_{k}(\delta \mid d)=0, \quad k>3 .
$$

Moreover, if the invariant polynomial $\alpha_{k}$, with agh $\left(\alpha_{k}\right)=k \geq 3$, is trivial in $H_{k}(\delta \mid d)$, then it can be taken to be trivial also in $H_{k}^{\text {inv }}(\delta \mid d)$

$$
\left(\alpha_{k}=\delta b_{k+1}+\partial_{\mu} \stackrel{(k)^{\mu}}{c}, \quad \operatorname{agh}\left(\alpha_{k}\right)=k \geq 3\right) \Rightarrow \alpha_{k}=\delta \beta_{k+1}+\partial_{\mu} \stackrel{(k)}{\gamma}^{\mu}
$$

where $\beta_{k+1}$ and $\stackrel{(k)}{\gamma}^{\mu}$ are invariant polynomials 4 . [An element of $H_{k}^{\text {inv }}(\delta \mid d)$

\footnotetext{
${ }^{3}$ We recall that the local cohomology $H(\delta \mid d)$ is completely trivial at both strictly positive antighost and pure ghost numbers (for instance, see [34, Theorem 5.4 or 35]).

${ }^{4}$ The proof can be realized in the same manner like Theorem 5 from [33, with the precaution to include in an appropriate manner the dependence on the vector field BRST sector.
} 
is defined via an equation similar to (44), but with the corresponding current an invariant polynomial.] The results (46) and (45) ensure that all the local cohomology of the Koszul-Tate differential in the space of invariant polynomials is trivial in antighost numbers strictly greater than three

$$
H_{k}^{\text {inv }}(\delta \mid d)=0, \quad k>3 .
$$

Using the definitions (18) and (20), we can organize the nontrivial representatives of $\left(H_{k}(\delta \mid d)\right)_{k \geq 2}$ and $\left(H_{k}^{\text {inv }}(\delta \mid d)\right)_{k \geq 2}$ like: i) for $k>3$ there are none; ii) for $k=3$ they are linear combinations of the undifferentiated antifields $C^{* \mu \nu}$ with constant coefficients; iii) for $k=2$ they are written like linear combinations of the undifferentiated antifields $\eta^{* \mu \nu \mid \alpha}$ and $\eta^{*}$ with constant coefficients. We have excluded from both $H(\delta \mid d)$ and $H^{\text {inv }}(\delta \mid d)$ the nontrivial elements depending on the space-time coordinates, as they would result in interactions with broken Poincaré invariance. In contrast to the groups $\left(H_{k}(\delta \mid d)\right)_{k \geq 2}$ and $\left(H_{k}^{\text {inv }}(\delta \mid d)\right)_{k \geq 2}$, which are finite-dimensional, the cohomology $H_{1}(\delta \mid d)$, that is related to global symmetries and ordinary conservation laws, is infinite-dimensional since the theory is free. Fortunately, it will not be needed in the sequel.

The previous results on $H(\delta \mid d)$ and $H^{\text {inv }}(\delta \mid d)$ at strictly positive antighost numbers are important because they control the obstructions to removing the antifields from the first-order deformation. As a consequence of the result (47), we can eliminate all the terms with $k>3$ from the expansion (30) by adding only trivial pieces and thus work with $I \leq 3$.

\subsection{Computation of the first-order deformation}

Now, we have at hand all the necessary ingredients for computing the general form of the first-order deformation of the solution to the master equation. In the case $I=3$ the nonintegrated density of the first-order deformation becomes

$$
a=a_{0}+a_{1}+a_{2}+a_{3} .
$$

We can further decompose $a$ in a natural manner as

$$
a=a^{\mathrm{t}}+a^{\mathrm{t}-\mathrm{A}}+a^{\mathrm{A}},
$$

where $a^{\mathrm{t}}$ contains only fields/ghosts/antifields from the $t_{\mu \nu \mid \alpha \beta}$ sector, $a^{\mathrm{t}-\mathrm{A}}$ describes the cross-interactions between the tensor field $t_{\mu \nu \mid \alpha \beta}$ the vector field 
(so it effectively mixes both sectors), and $a^{\mathrm{A}}$ involves only the vector field sector. As it has been shown in [36] under the hypotheses of smoothness, locality, Lorentz covariance, and Poincaré invariance of the deformations, combined with the requirement that the interacting Lagrangian is at most second-order derivative, to be maintained here as well, $a^{\mathrm{t}}$ satisfies an equation similar to (29) and has the expression

$$
a^{\mathrm{t}}=c^{\prime} t \equiv t_{\mid \mu \nu}^{\mu \nu}
$$

with $c^{\prime}$ an arbitrary, real constant. On the other hand $a^{\mathrm{t}-\mathrm{A}}$ and $a^{\mathrm{A}}$ involve different sorts of fields, so these components verify independently some equations similar to (29)

$$
\begin{aligned}
s a^{\mathrm{t}-\mathrm{A}} & =\partial_{\mu} m^{(\mathrm{t}-\mathrm{A}) \mu} \\
s a^{\mathrm{A}} & =\partial_{\mu} m^{(\mathrm{A}) \mu},
\end{aligned}
$$

for some local $m^{\mu}$ 's. In the sequel we analyze the general solutions to these equations.

The term $a^{\mathrm{t}-\mathrm{A}}$ allows a decomposition similar to (48)

$$
a^{\mathrm{t}-\mathrm{A}}=a_{0}^{\mathrm{t}-\mathrm{A}}+a_{1}^{\mathrm{t}-\mathrm{A}}+a_{2}^{\mathrm{t}-\mathrm{A}}+a_{3}^{\mathrm{t}-\mathrm{A}},
$$

where the components of $a^{\mathrm{t}-\mathrm{A}}$ are subject to the equations

$$
\begin{aligned}
\gamma a_{3}^{\mathrm{t}-\mathrm{A}} & =0 \\
\delta a_{I}^{\mathrm{t}-\mathrm{A}}+\gamma a_{I-1}^{\mathrm{t}-\mathrm{A}} & =\partial_{\mu}{ }^{(I-1)}{ }^{(\mathrm{t}-\mathrm{A}) \mu}, \quad I=1,2,3 .
\end{aligned}
$$

In agreement with (43) and with the discussion made in the above regarding the nontrivial representatives of $H_{3}^{\text {inv }}(\delta \mid d)$ (see the case ii)) the equation (54) possesses in $D \geq 5$ space-time dimensions the solution

$$
a_{3}^{\mathrm{t}-\mathrm{A}}=a_{3}^{(1) \mathrm{t}-\mathrm{A}}+a_{3}^{(2) \mathrm{t}-\mathrm{A}}
$$

where:

- for all $D \geq 5$

$$
a_{3}^{(1) \mathrm{t}-\mathrm{A}}=c_{1} C^{* \mu \nu} C_{\mu \nu} \eta ;
$$

- for $D=5$

$$
a_{3}^{(2) \mathrm{t}-\mathrm{A}}=c_{2} \varepsilon^{\mu \nu \lambda \beta \rho} C_{\mu \nu}^{*}\left(\partial_{\lambda} C_{\beta \rho}\right) \eta .
$$


In the relations (57)-(58) $c_{1}$ and $c_{2}$ are some arbitrary, real constants. Obviously, since the components (57)-(58) are mutually independent, it follows that each of them must separately fulfill an equation of type (55) for $I=3$

$$
\delta a_{3}^{(i) \mathrm{t}-\mathrm{A}}=-\gamma a_{2}^{(i) \mathrm{t}-\mathrm{A}}+\partial_{\mu}{ }^{(2)}{ }^{(i)(\mathrm{t}-\mathrm{A}) \mu}, \quad i=1,2 .
$$

By direct computation we obtain

$$
\begin{aligned}
\delta a_{3}^{(1) \mathrm{t}-\mathrm{A}}= & -\gamma\left[c_{1} \eta^{* \mu \nu \mid \alpha}\left(\frac{3}{2} \eta_{\mu \nu \mid \alpha} \eta-C_{\mu \nu} A_{\alpha}\right)\right]+\partial_{\mu} u^{\mu} \\
& +\frac{3}{2} c_{1} \eta^{* \mu \nu \mid \alpha} \partial_{[\mu} C_{\nu] \alpha} \eta .
\end{aligned}
$$

Thus, $a_{3}^{(1) \mathrm{t}-\mathrm{A}}$ produces a consistent $a_{2}^{(1) \mathrm{t}-\mathrm{A}}$ as solution to the equation (59) for $i=1$ if and only if the term $(3 / 2) c_{1} \eta^{* \mu \nu \mid \alpha} \partial_{[\mu} C_{\nu] \alpha} \eta$, which is a nontrivial representative of $H(\gamma)$, is written in a $\gamma$-exact modulo $d$ form. This takes place if and only if

$$
c_{1}=0 .
$$

Related to $a_{3}^{(2) \mathrm{t}-\mathrm{A}}$, by applying $\delta$ on (58) we find via the equation (599) for $i=2$ that $a_{2}^{(2) \mathrm{t}-\mathrm{A}}$ reads as

$$
\begin{aligned}
a_{2}^{(2) \mathrm{t}-\mathrm{A}}= & 3 c_{2} \varepsilon^{\mu \nu \lambda \beta \rho} \eta_{\mu \nu \mid}^{* \alpha}\left[\left(\frac{5}{12} \partial_{\lambda} \eta_{\beta \rho \mid \alpha}+\frac{1}{6} \partial_{\beta} \eta_{\rho \alpha \mid \lambda}\right) \eta\right. \\
& \left.-\left(\partial_{\lambda} C_{\beta \rho}\right) A_{\alpha}\right] .
\end{aligned}
$$

By means of (62) we deduce

$$
\begin{aligned}
\delta a_{2}^{(2) \mathrm{t}-\mathrm{A}}= & \partial_{\mu} v^{\mu}-\gamma\left[c _ { 2 } \varepsilon ^ { \mu \nu \lambda \beta \rho } t ^ { * \tau \alpha | } { } _ { \mu \nu } \left(3\left(\partial_{\lambda} t_{\tau \alpha \mid \beta \rho}\right) \eta\right.\right. \\
& \left.\left.+\left(5 \partial_{\lambda} \eta_{\beta \rho \mid \tau}+2 \partial_{\beta} \eta_{\rho \tau \mid \lambda}\right) A_{\alpha}\right)\right] \\
& -6 c_{2} \varepsilon^{\mu \nu \lambda \beta \rho} t^{* \tau \alpha \mid}{ }_{\mu \nu}\left(\partial_{\lambda} C_{\beta \rho}\right) F_{\tau \alpha} .
\end{aligned}
$$

Comparing (63) with (551) for $I=2$ we can state that $a_{2}^{(2) t-A}$ provides a consistent $a_{1}^{(2) \mathrm{t}-\mathrm{A}}$ if and only if the term $6 c_{2} \varepsilon^{\mu \nu \lambda \beta \rho} t^{* \tau \alpha \mid}{ }_{\mu \nu}\left(\partial_{\lambda} C_{\beta \rho}\right) F_{\tau \alpha}$, which is again a nontrivial representative of $H(\gamma)$, is $\gamma$-exact modulo $d$. This holds if and only if

$$
c_{2}=0 .
$$

The results (61) and (64) show that

$$
a_{3}^{\mathrm{t}-\mathrm{A}}=0 .
$$


Accordingly, $a^{\mathrm{t}-\mathrm{A}}$ can stop earliest at antighost number two

$$
a^{\mathrm{t}-\mathrm{A}}=a_{0}^{\mathrm{t}-\mathrm{A}}+a_{1}^{\mathrm{t}-\mathrm{A}}+a_{2}^{\mathrm{t}-\mathrm{A}},
$$

with $a_{2}^{\mathrm{t}-\mathrm{A}}$ solution to the equation $\gamma a_{2}^{\mathrm{t}-\mathrm{A}}=0$. Looking at (43) for $I=2$, using the previous results on the nontrivial representatives of $H_{2}^{\text {inv }}(\delta \mid d)$ (see the case iii) in the above), and requiring that $a_{2}^{\mathrm{t}-\mathrm{A}}$ effectively describes crosscouplings, we get (up to trivial, $\gamma$-exact contributions) that

$$
a_{2}^{\mathrm{t}-\mathrm{A}}=\eta^{*}\left(\lambda^{\mu \nu} C_{\mu \nu}+\bar{\lambda}^{\mu \nu \alpha} \partial_{[\mu} C_{\nu] \alpha}\right),
$$

where $\lambda^{\mu \nu}$ and $\bar{\lambda}^{\mu \nu \alpha}$ are some non-derivative, real constant tensors, invariant under the Lorentz group, with $\lambda^{\mu \nu}$ and $\bar{\lambda}^{\mu \nu \alpha}$ antisymmetric in $\lambda$ and $\mu$. Since in $D \geq 5$ there are no such constants we must set $\lambda^{\mu \nu}=0$ and $\bar{\lambda}^{\mu \nu \alpha}=0$, so we have that

$$
a_{2}^{\mathrm{t}-\mathrm{A}}=0 .
$$

In this way we infer that $a^{\mathrm{t}-\mathrm{A}}$ actually stops at antighost number one

$$
a^{\mathrm{t}-\mathrm{A}}=a_{0}^{\mathrm{t}-\mathrm{A}}+a_{1}^{\mathrm{t}-\mathrm{A}},
$$

with $a_{1}^{\mathrm{t}-\mathrm{A}}$ solution to the equation $\gamma a_{1}^{\mathrm{t}-\mathrm{A}}=0$. Since $a_{1}^{\mathrm{t}-\mathrm{A}}$ is linear in the antifields of the original fields, we can write

$$
a_{1}^{\mathrm{t}-\mathrm{A}}=\left(t_{\mu \nu \mid \alpha \beta}^{*} \Delta^{\mu \nu \mid \alpha \beta}+A_{\mu}^{*} \Delta^{\mu}\right) \eta,
$$

where $\Delta^{\mu \nu \mid \alpha \beta}$ and $\Delta^{\mu}$ are $\gamma$-invariant objects (with both the antighost number and the pure ghost number equal to zero), the former quantities displaying the mixed symmetry of the tensor $t^{\mu \nu \mid \alpha \beta}$. From (43) at antighost number zero we observe that $\Delta^{\mu \nu \mid \alpha \beta}$ and $\Delta^{\mu}$ depend in general on $F^{\mu \nu}, F^{\mu \nu \lambda \mid \alpha \beta \gamma}$, and their derivatives. Moreover, the requirement that the second term in the right-hand side of (70) produces cross-interactions implies that $\Delta^{\mu}$ must involve $F^{\mu \nu \lambda \mid \alpha \beta \gamma}$ (and possibly its derivatives). In order to construct $a_{0}^{\mathrm{t}-\mathrm{A}}$ as solution to the equation

$$
\delta a_{1}^{\mathrm{t}-\mathrm{A}}+\gamma a_{0}^{\mathrm{t}-\mathrm{A}}=\partial_{\mu} \stackrel{(0)}{(\mathrm{t}-\mathrm{A}) \mu}^{,}
$$

we invoke the hypothesis on the maximum derivative order of $a_{0}^{\mathrm{t}-\mathrm{A}}$ being equal to two. As both $\delta t_{\mu \nu \mid \alpha \beta}^{*}$ and $\delta A_{\mu}^{*}$ contain exactly two derivatives, it follows that each of $\Delta^{\mu \nu \mid \alpha \beta}$ and $\Delta^{\mu}$ are allowed to include at most one derivative (we remind that $\partial_{\mu} \eta=\gamma A_{\mu}$ ) and therefore we have that

$$
\Delta^{\mu \nu \mid \alpha \beta}=\Delta_{0}^{\mu \nu \mid \alpha \beta}+\Delta_{1}^{\mu \nu \mid \alpha \beta}, \quad \Delta^{\mu}=\Delta_{0}^{\mu}+\Delta_{1}^{\mu},
$$


where $\Delta_{0}$ 's contain no derivatives and $\Delta_{1}$ 's include just one derivative. Therefore, both $\Delta_{0}$ 's must be constant, while $\Delta_{1}$ 's must depend linearly on $F_{\mu \nu}$. From covariance arguments in $D \geq 5$ we have that the only possible choice of these quantities is

$$
\Delta_{0}^{\mu \nu \mid \alpha \beta}=\frac{1}{2} k_{1}\left(\sigma^{\mu \alpha} \sigma^{\nu \beta}-\sigma^{\mu \beta} \sigma^{\nu \alpha}\right), \quad \Delta_{0}^{\mu}=0,
$$

and

$$
\Delta_{1}^{\mu \nu \mid \alpha \beta}=k_{2} \varepsilon^{\mu \nu \alpha \beta \lambda \rho} F_{\lambda \rho}, \quad \Delta_{1}^{\mu}=0,
$$

with $k_{1}$ and $k_{2}$ arbitrary, real constants. The solution (74) 'lives' in $D=6$, but it brings no contribution to $a_{1}^{\mathrm{t}-\mathrm{A}}$ as $\varepsilon^{\mu \nu \alpha \beta \lambda \rho} t_{\mu \nu \mid \alpha \beta}^{*} \equiv 0$. Substituting (72) $-(74)$ in (70) we find that

$$
a_{1}^{\mathrm{t}-\mathrm{A}}=k_{1} t^{*} \eta
$$

By applying $\delta$ on (75) we deduce

$$
\delta a_{1}^{\mathrm{t}-\mathrm{A}}=\frac{k_{1}}{4}(4-D)(3-D)\left(\partial^{\mu} \partial^{\rho} t_{\mu \rho}-\frac{1}{2} \square t\right) \eta .
$$

In order to analyze the solution to the equation (171), we assume that $a_{1}^{\mathrm{t}-\mathrm{A}}$ of the form (75) generates a consistent $a_{0}^{\mathrm{t}-\mathrm{A}}$. From (76) it follows that the corresponding $a_{0}^{\mathrm{t}-\mathrm{A}}$ is linear in both $t_{\mu \nu \mid \alpha \beta}$ and $A_{\mu}$ and contains precisely one space-time derivative. Then, up to an irrelevant divergence, $a_{0}^{\mathrm{t}-\mathrm{A}}$ reads as

$$
a_{0}^{\mathrm{t}-\mathrm{A}}=A_{\mu} m^{\mu}(\partial t)
$$

where $m^{\mu}(\partial t)$ is linear in the first-order derivatives of $t_{\mu \nu \mid \alpha \beta}$. It is simple to see that the most general form of $m^{\mu}(\partial t)$ can be represented like

$$
m^{\mu}(\partial t)=c_{3} \partial^{\mu} t+c_{4} \partial_{\rho} t^{\mu \rho}
$$

with $c_{3}$ and $c_{4}$ arbitrary, real constants, such that

$$
a_{0}^{\mathrm{t}-\mathrm{A}}=A_{\mu}\left(c_{3} \partial^{\mu} t+c_{4} \partial_{\rho} t^{\mu \rho}\right) .
$$

By direct computation, from (76) and (79) we obtain

$$
\delta a_{1}^{\mathrm{t}-\mathrm{A}}+\gamma a_{0}^{\mathrm{t}-\mathrm{A}}=\partial_{\mu}\left[\frac{k_{1}}{4}(4-D)(3-D)\left(\partial_{\rho} t^{\mu \rho}-\frac{1}{2} \partial^{\mu} t\right) \eta\right.
$$




$$
\begin{aligned}
& +\left(4 c_{3}+c_{4}\right) A^{\mu} \partial^{\rho} \eta_{\rho \beta \mid}{ }^{\beta} \\
& \left.+c_{4} A^{\alpha}\left(\partial^{\mu} \eta_{\alpha \beta \mid}{ }^{\beta}-\partial^{\beta} \eta_{\alpha \beta \mid}{ }^{\mu}\right)\right] \\
& +\left(c_{3}+\frac{k_{1}}{8}(4-D)(3-D)\right)\left(\partial^{\mu} t\right) \partial_{\mu} \eta \\
& +\left(c_{4}-\frac{k_{1}}{4}(4-D)(3-D)\right)\left(\partial_{\rho} t^{\mu \rho}\right) \partial_{\mu} \eta \\
& -\left(4 c_{3}+c_{4}\right)\left(\partial_{\mu} A^{\mu}\right) \partial^{\rho} \eta_{\rho \beta \mid}{ }^{\beta}{ }^{\mu} \\
& -c_{4}\left(\partial_{\mu} A^{\alpha}\right)\left(\partial^{\mu} \eta_{\alpha \beta \mid}{ }^{\beta}-\partial^{\beta} \eta_{\alpha \beta \mid}\right) .
\end{aligned}
$$

The right-hand side of (80) reduces to a total derivative (as it is required by the equation (71)) if and only if the constants $k_{1}, c_{3}$, and $c_{4}$ satisfy the equations

$$
\begin{aligned}
c_{3}+\frac{k_{1}}{8}(4-D)(3-D) & =0, \\
c_{4}-\frac{k_{1}}{4}(4-D)(3-D) & =0, \\
4 c_{3}+c_{4} & =0, \\
c_{4} & =0,
\end{aligned}
$$

allowing only the vanishing solution

$$
k_{1}=c_{3}=c_{4}=0 \text {. }
$$

As a consequence, we find that

$$
a_{1}^{\mathrm{t}-\mathrm{A}}=0
$$

so $a^{\mathrm{t}-\mathrm{A}}$ actually reduces to its component of antighost number zero,

$$
a^{\mathrm{t}-\mathrm{A}}=a_{0}^{\mathrm{t}-\mathrm{A}}
$$

which is subject to the 'homogeneous' equation

$$
\gamma a_{0}^{\mathrm{t}-\mathrm{A}}=\partial_{\mu} \stackrel{(0)}{m}^{(\mathrm{t}-\mathrm{A}) \mu}
$$


There are two main types of solutions to this equation. The first type, to be denoted by $a_{0}^{\text {tt-A }}$, corresponds to $\stackrel{(0)}{m}^{(\mathrm{t}-\mathrm{A}) \mu}=0$ and is given by gaugeinvariant, nonintegrated densities constructed out of the original fields and their space-time derivatives, which, according to (43), are of the form

$$
a_{0}^{\prime \mathrm{t}-\mathrm{A}}=a_{0}^{\prime \mathrm{t}-\mathrm{A}}\left(\left[F_{\mu \nu}\right],\left[F_{\mu \nu \lambda \mid \alpha \beta \gamma}\right]\right)
$$

up to the condition that they effectively describe cross-couplings between the two types of fields and cannot be written in a divergence-like form. Such a solution would produce vertices with more than two derivatives of the fields and must be excluded since this disagrees with the hypothesis on the maximum derivative order 5

$$
a_{0}^{\text {t- }-\mathrm{A}}=0
$$

The second kind of solutions, to be denoted by $a_{0}^{\prime \prime t-A}$, is associated with ${ }_{(0)}^{(\mathrm{t}-\mathrm{A}) \mu}$

$\stackrel{(0)}{m}^{(t-A) \mu} \neq 0$ in ( $(\underline{88})$, being understood that we discard the divergence-like quantities and maintain the condition on the maximum derivative order. In order to solve the equation

$$
\gamma a_{0}^{\prime \prime \mathrm{t}-\mathrm{A}}=\partial_{\mu} \stackrel{(0)}{(\mathrm{t}-\mathrm{A}) \mu}^{,}
$$

we start from the requirement that $a_{0}^{\prime \prime t-A}$ may contain at most two derivatives. Then, $a_{0}^{\prime \prime t-A}$ can be decomposed like

$$
a_{0}^{\prime \prime \mathrm{t}-\mathrm{A}}=\omega_{0}+\omega_{1}+\omega_{2}
$$

where $\left(\omega_{i}\right)_{i=\overline{0,2}}$ contains $i$ derivatives. Due to the different number of derivatives in the components $\omega_{0}, \omega_{1}$, and $\omega_{2}$, the equation (91) leads to three independent equations

$$
\gamma \omega_{k}=\partial_{\mu} j_{k}^{\mu}, \quad k=0,1,2 .
$$

For $k=0$ the equation (93) implies the necessary conditions $\partial_{\mu}\left(\partial \omega_{0} / \partial t_{\mu \nu \mid \alpha \beta}\right)=$ 0 and $\partial_{\mu}\left(\partial \omega_{0} / \partial A_{\mu}\right)=0$, whose solutions read as $\partial \omega_{0} / \partial t_{\mu \nu \mid \alpha \beta}=k^{\mu \nu \mid \alpha \beta}$ and $\partial \omega_{0} / \partial A_{\mu}=k^{\mu}$, where $k^{\mu \nu \mid \alpha \beta}$ and $k^{\mu}$ are arbitrary, real constants. The last

\footnotetext{
${ }^{5}$ If we however relax the derivative-order condition, then we can find nonvanishing solutions of the type (89). An example of a possible solution is represented by the cubic vertex $a_{0}^{\text {tt-A }}=F_{\mu \nu \lambda \mid \alpha \beta \gamma} F^{\mu \nu} F^{\alpha \beta} \sigma^{\lambda \gamma}$.
} 
solutions provide $\omega_{0}=k^{\mu \nu \mid \alpha \beta} t_{\mu \nu \mid \alpha \beta}+k^{\mu} A_{\mu}$, so it does not describe crossinteractions between $t_{\mu \nu \mid \alpha \beta}$ and $A_{\mu}$ and can be made to vanish, $\omega_{0}=0$. For $k=1$ the equation (93) requires that

$$
\partial_{\mu} \frac{\delta \omega_{1}}{\delta t_{\mu \nu \mid \alpha \beta}}=0, \quad \partial_{\mu} \frac{\delta \omega_{1}}{\delta A_{\mu}}=0
$$

whose solutions are of the type 6

$$
\frac{\delta \omega_{1}}{\delta t_{\mu \nu \mid \alpha \beta}}=0, \quad \frac{\delta \omega_{1}}{\delta A_{\mu}}=\partial_{\nu} B^{\mu \nu}
$$

where $\delta \omega_{1} / \delta t_{\mu \nu \mid \alpha \beta}$ and $\delta \omega_{1} / \delta A_{\mu}$ denote the variational derivatives of $\omega_{1}$. In the above the antisymmetric functions $B^{\mu \nu}$ have no derivatives. Using (95), we conclude that, up to an irrelevant divergence, $\omega_{1}$ is a functions of $A_{\mu}$ with precisely one derivative. Such an $\omega_{1}$ does not provide cross-interactions between $t_{\mu \nu \mid \alpha \beta}$ and $A_{\mu}$, so we can take $\omega_{1}=0$.

In the sequel we consider the equation (93) for $k=2$, which gives the necessary conditions

$$
\partial_{\mu} \frac{\delta \omega_{2}}{\delta t_{\mu \nu \mid \alpha \beta}}=0, \quad \partial_{\mu} \frac{\delta \omega_{2}}{\delta A_{\mu}}=0
$$

with the solutions

$$
\frac{\delta \omega_{2}}{\delta t_{\mu \nu \mid \alpha \beta}}=\partial_{\lambda} \partial_{\rho} U^{\mu \nu \lambda \mid \alpha \beta \rho}, \quad \frac{\delta \omega_{2}}{\delta A_{\mu}}=\partial_{\nu} \Phi^{\mu \nu} .
$$

Let $N$ be a derivation in the algebra of the fields and of their derivatives that counts the powers of the fields and their derivatives, defined by

$$
\begin{aligned}
N= & \sum_{k \geq 0}\left(\left(\partial_{\mu_{1}} \cdots \partial_{\mu_{k}} t_{\mu \nu \mid \alpha \beta}\right) \frac{\partial}{\partial\left(\partial_{\mu_{1}} \cdots \partial_{\mu_{k}} t_{\mu \nu \mid \alpha \beta}\right)}\right. \\
& \left.+\left(\partial_{\mu_{1}} \cdots \partial_{\mu_{k}} A_{\mu}\right) \frac{\partial}{\partial\left(\partial_{\mu_{1}} \cdots \partial_{\mu_{k}} A_{\mu}\right)}\right) .
\end{aligned}
$$

\footnotetext{
${ }^{6}$ In general, the solution to the equation $\partial_{\mu}\left(\delta \alpha / \delta t_{\mu \nu \mid \alpha \beta}\right)=0$ has the form $\delta \alpha / \delta t_{\mu \nu \mid \alpha \beta}=$ $\partial_{\lambda} \partial_{\rho} M^{\mu \nu \lambda \mid \alpha \beta \rho}+m^{\mu \nu \mid \alpha \beta}$, where the functions $M^{\mu \nu \lambda \mid \alpha \beta \rho}$ display the mixed symmetry of the curvature tensor and $m^{\mu \nu \mid \alpha \beta}$ are some non-derivative constants with the mixed symmetry $(2,2)$. If we ask that $\alpha$ comprises one space-time derivative, then we must set $M^{\mu \nu \lambda \mid \alpha \beta \rho}=0$ and $m^{\mu \nu \mid \alpha \beta}=0$, which justifies the former solution from (95).
} 
Then, it is easy to see that for every nonintegrated density $\chi$, we have that

$$
N \chi=t_{\mu \nu \mid \alpha \beta} \frac{\delta \chi}{\delta t_{\mu \nu \mid \alpha \beta}}+A_{\mu} \frac{\delta \chi}{\delta A_{\mu}}+\partial_{\mu} s^{\mu} .
$$

If $\chi^{(l)}$ is a homogeneous polynomial of order $l>0$ in the fields $\left\{t_{\mu \nu \mid \alpha \beta}, A_{\mu}\right\}$ and their derivatives, then $N \chi^{(l)}=l \chi^{(l)}$. On account of (97) and (99), we find that

$$
N \omega_{2}=\frac{1}{9} F_{\mu \nu \lambda \mid \alpha \beta \rho} U^{\mu \nu \lambda \mid \alpha \beta \rho}+\frac{1}{2} F_{\mu \nu} \Phi^{\mu \nu}+\partial_{\mu} v^{\mu} .
$$

We expand $\omega_{2}$ like

$$
\omega_{2}=\sum_{l>0} \omega_{2}^{(l)}
$$

where $N \omega_{2}^{(l)}=l \omega_{2}^{(l)}$, such that

$$
N \omega_{2}=\sum_{l>0} l \omega_{2}^{(l)}
$$

Comparing (100) with (102), we reach the conclusion that the decomposition (101) induces a similar decomposition with respect to $U^{\mu \nu \lambda \mid \alpha \beta \rho}$ and $\Phi^{\mu \nu}$, i.e.

$$
U^{\mu \nu \lambda \mid \alpha \beta \rho}=\sum_{l>0} U_{(l-1)}^{\mu \nu \lambda \mid \alpha \beta \rho}, \quad \Phi^{\mu \nu}=\sum_{l>0} \Phi_{(l-1)}^{\mu \nu}
$$

Substituting (103) into (100) and comparing the resulting expression with (102), we obtain that

$$
\omega_{2}^{(l)}=\frac{1}{9 l} F_{\mu \nu \lambda \mid \alpha \beta \rho} U_{(l-1)}^{\mu \nu \lambda \mid \alpha \beta \rho}+\frac{1}{2 l} F_{\mu \nu} \Phi_{(l-1)}^{\mu \nu}+\partial_{\mu} \bar{v}_{(l)}^{\mu} .
$$

Introducing (104) in (101), we arrive at

$$
\omega_{2}=F_{\mu \nu \lambda \mid \alpha \beta \rho} \bar{U}^{\mu \nu \lambda \mid \alpha \beta \rho}+F_{\mu \nu} \bar{\Phi}^{\mu \nu}+\partial_{\mu} \bar{v}^{\mu},
$$

where

$$
\bar{U}^{\mu \nu \lambda \mid \alpha \beta \rho}=\sum_{l>0} \frac{1}{9 l} U_{(l-1)}^{\mu \nu \lambda \mid \alpha \beta \rho}, \quad \bar{\Phi}^{\mu \nu}=\sum_{l>0} \frac{1}{2 l} \Phi_{(l-1)}^{\mu \nu} .
$$

By applying $\gamma$ on the relation (105), after long and tedious computation we infer that a necessary condition for the existence of solutions to the equation $\gamma \omega_{2}=\partial_{\mu} j_{2}^{\mu}$ is that the functions $\bar{U}^{\mu \nu \lambda \mid \alpha \beta \rho}$ and $\bar{\Phi}^{\mu \nu}$ have the expressions

$$
\bar{U}^{\mu \nu \lambda \mid \alpha \beta \rho}=C^{\mu \nu \lambda \mid \alpha \beta \rho ; \sigma} A_{\sigma}, \quad \bar{\Phi}^{\mu \nu}=\bar{k}^{\mu \nu \rho ; \alpha \beta \mid \sigma \lambda} \partial_{\rho} t_{\alpha \beta \mid \sigma \lambda},
$$


where $C^{\mu \nu \lambda \mid \alpha \beta \rho ; \sigma}$ and $\bar{k}^{\mu \nu \rho ; \alpha \beta \mid \sigma \lambda}$ are non-derivative, real constants. The former constants exhibit the mixed symmetry $(3,3)$ in the indices $\mu \nu \lambda \mid \alpha \beta \rho$ and are separately antisymmetric in $\{\alpha, \beta, \rho, \sigma\}$. The quantities $\bar{k}^{\mu \nu \rho ; \alpha \beta \mid \sigma \lambda}$ are antisymmetric in the indices $\{\mu, \nu, \rho\}$ and display the mixed symmetry $(2,2)$ with respect to $\alpha \beta \mid \sigma \lambda$. Substituting (107) in (105) we get that

$$
\omega_{2}=C^{\mu \nu \lambda \mid \alpha \beta \rho ; \sigma} F_{\mu \nu \lambda \mid \alpha \beta \rho} A_{\sigma}+\partial_{\rho}\left(F_{\mu \nu} \bar{k}^{\mu \nu \rho ; \alpha \beta \mid \sigma \lambda} t_{\alpha \beta \mid \sigma \lambda}+\bar{v}^{\rho}\right) .
$$

As a consequence, the existence of a nontrivial $\omega_{2}$ is conditioned by the existence of some pure constants $C^{\mu \nu \lambda \mid \alpha \beta \rho ; \sigma}$ that must simultaneously display the mixed symmetry $(3,3)$ in their first six indices and be antisymmetric in the indices $\{\alpha, \beta, \rho, \sigma\}$. Because of the odd number of indices in $C^{\mu \nu \lambda \mid \alpha \beta \rho ; \sigma}$, these constants can only be constructed from the flat metric $\sigma^{\mu \nu}$ and LeviCivita symbols $\varepsilon^{\mu_{1} \cdots \mu_{j}}$. Due to the identity $F_{[\mu \nu \lambda \mid \alpha] \beta \rho} \equiv 0$, the Levi-Civita symbols can be contracted with $F_{\mu \nu \lambda \mid \alpha \beta \rho}$ on at most three indices. On the other hand, the restriction $D \geq 5$ on the space-time dimension requires LeviCivita symbols with at least five indices, so $\varepsilon^{\mu_{1} \cdots \mu_{j}}$ will contract with $F_{\mu \nu \lambda \mid \alpha \beta \rho}$ on at least four indices, such that the corresponding $\omega_{2}$ will vanish identically. In consequence, we can take

$$
C^{\mu \nu \lambda \mid \alpha \beta \rho ; \sigma}=0
$$

which further leads to

$$
a_{0}^{\prime \prime \mathrm{t}-\mathrm{A}}=0
$$

The relations (90) and (109) show that

$$
a_{0}^{\mathrm{t}-\mathrm{A}}=0 .
$$

By means of the results (53), (65), (68), (6ㅜ), and (110) we arrive at

$$
a^{\mathrm{t}-\mathrm{A}}=0 .
$$

Finally, we focus on the solutions to the equation (52). It is easy to see that $a^{\mathrm{A}}$ can only reduce to its component of antighost number zero

$$
a^{\mathrm{A}}=a_{0}^{\mathrm{A}}\left(\left[A_{\mu}\right]\right),
$$

which is solution to the equation $s a^{\mathrm{A}} \equiv \gamma a_{0}^{\mathrm{A}}=\partial_{\mu} m_{0}^{(\mathrm{A}) \mu}$. It comes from $a_{1}^{\mathrm{A}}=0$ and does not deform the gauge transformations, but merely modifies 
the vector field action. The condition that $a_{0}^{\mathrm{A}}$ is of maximum derivative order equal to two is translated into

$$
a_{0}^{\mathrm{A}}=c^{\prime \prime} \varepsilon^{\mu \nu \lambda \beta \rho} A_{\mu} F_{\nu \lambda} F_{\beta \rho},
$$

for $D=5$, with $c^{\prime \prime}$ an arbitrary, real constant. Putting together the results deduced so far, we obtained that the first-order deformation of the solution to the master equation for the theory (1) has the expression

$$
a=c^{\prime} t+c^{\prime \prime} \varepsilon^{\mu \nu \lambda \beta \rho} A_{\mu} F_{\nu \lambda} F_{\beta \rho} .
$$

\subsection{Higher-order deformations}

Taking into account the equations (28), etc., we get that the first-order deformation (114) is consistent to all orders in the coupling constant. Indeed, as $\left(S_{1}, S_{1}\right)=0$, the equation (28), which describes the second-order deformation, is satisfied with the choice

$$
S_{2}=0
$$

while the remaining higher-order equations are fulfilled for

$$
S_{3}=S_{4}=\cdots=0 .
$$

The fact that $a^{\mathrm{t}-\mathrm{A}}=0$ shows there are no consistent cross-couplings between the massless tensor field $t_{\mu \nu \mid \alpha \beta}$ and the vector field $A_{\mu}$ complying with all the hypotheses used in this paper.

\section{Conclusion}

To conclude with, in this paper we have investigated the couplings between the massless tensor field with the mixed symmetry of the Riemann tensor and the massless vector field by using the powerful setting based on local BRST cohomology. Under the assumptions on smoothness, locality, Lorentz covariance, and Poincare invariance of the deformations, combined with the requirement that the interacting Lagrangian is at most second-order derivative, we have proved that there are no consistent cross-interactions between such fields. Our approach opens the perspective of investigating the interactions between the tensor field $t_{\mu \nu \mid \alpha \beta}$ and one $p$-form $(p>1)$ or, more general, between a tensor field with the mixed symmetry $(k, k)$ and a $p$-form. These problems are under consideration. 


\section{Acknowledgment}

The authors are partially supported by the European Commission FP6 program MRTN-CT-2004-005104 and by the type A grant 304/2004 with the Romanian National Council for Academic Scientific Research and the Romanian Ministry of Education and Research.

\section{References}

[1] T. Curtright, Phys. Lett. B165 (1985) 304

[2] T. Curtright, P. G. O. Freund, Nucl. Phys. B172 (1980) 413

[3] C. S. Aulakh, I. G. Koh, S. Ouvry, Phys. Lett. B173 (1986) 284

[4] J. M. Labastida, T. R. Morris, Phys. Lett. B180 (1986) 101

[5] J. M. Labastida, Nucl. Phys. B322 (1989) 185

[6] C. Burdik, A. Pashnev, M. Tsulaia, Mod. Phys. Lett. A16 (2001) 731

[7] Yu. M. Zinoviev, hep-th/0211233

[8] C. M. Hull, JHEP 0109 (2001) 027

[9] X. Bekaert, N. Boulanger, Class. Quantum Grav. 20 (2003) S417

[10] X. Bekaert, N. Boulanger, Phys. Lett. B561 (2003) 183

[11] X. Bekaert, N. Boulanger, Commun. Math. Phys. 245 (2004) 27

[12] N. Boulanger, S. Cnockaert, M. Henneaux, JHEP 0306 (2003) 060

[13] H. Casini, R. Montemayor, L. F. Urrutia, Phys. Lett. B507 (2001) 336

[14] H. Casini, R. Montemayor, L. F. Urrutia, Phys. Rev. D68 (2003) 065011

[15] P. de Medeiros, C. Hull, Commun. Math. Phys. 235 (2003) 255

[16] X. Bekaert, N. Boulanger, M. Henneaux, Phys. Rev. D67 (2003) 044010

[17] Yu. M. Zinoviev, hep-th/0304067 
[18] Yu. M. Zinoviev, hep-th/0306292

[19] A. K. Bengtsson, I. Bengtsson, L. Brink, Nucl. Phys. B227 (1983) 41

[20] M. A. Vasiliev, Nucl. Phys. B616 (2001) 106; Erratum-ibid. B652 (2003) 407

[21] E. Sezgin, P. Sundell, Nucl. Phys. B634 (2002) 120

[22] D. Francia, A. Sagnotti, Phys. Lett. B543 (2002) 303

[23] C. Bizdadea, C. C. Ciobirca, E. M. Cioroianu, I. Negru, S. O. Saliu, S. C. Sararu, JHEP 0310 (2003) 019

[24] X. Bekaert, N. Boulanger, S. Cnockaert, J. Math. Phys. 46 (2005) 012303

[25] N. Boulanger, S. Cnockaert, JHEP 0403 (2004) 031

[26] C. C. Ciobîrcă, E. M. Cioroianu, S. O. Saliu, Int. J. Mod. Phys. A19 (2004) 4579

[27] N. Boulanger, S. Leclercq, S. Cnockaert, Phys.Rev. D73 (2006) 065019

[28] X. Bekaert, N. Boulanger, S. Cnockaert, JHEP 0601 (2006) 052

[29] G. Barnich, M. Henneaux, Phys. Lett. B311 (1993) 123

[30] M. Henneaux, Contemp. Math. 219 (1998) 93

[31] G. Barnich, F. Brandt, M. Henneaux, Commun. Math. Phys. 174 (1995) 93

[32] G. Barnich, M. Henneaux, Phys. Rev. Lett. 72 (1994) 1588

[33] C. Bizdadea, C. C. Ciobîrcă, E. M. Cioroianu, S. O. Saliu, S. C. Săraru, Int. J. Geom. Meth. Mod. Phys. 1 (2004) 335

[34] G. Barnich, F. Brandt, M. Henneaux, Commun. Math. Phys. 174 (1995) 57

[35] G. Barnich, F. Brandt, M. Henneaux, Phys. Rept. 338 (2000) 439

[36] C. Bizdadea, C. C. Ciobîrcă, E. M. Cioroianu, S. O. Saliu, S. C. Săraru, Eur. Phys. J. C36 (2004) 253 